\title{
Layer-by-layer Deposition of Organically Capped Quantum Dots
}

\author{
Márcia C. Neves ${ }^{1, a}$, Angela S. Pereira ${ }^{1, b}$, Marco Peres ${ }^{2, c}$, Andrei Kholkin ${ }^{3, d}$, \\ Teresa Monteiro $^{2, \mathrm{e}}$, Tito Trindade ${ }^{1, \mathrm{f}}$ \\ ${ }^{1}$ Department of Chemistry, University of Aveiro, CICECO, 3810-193 Aveiro, Portugal \\ ${ }^{2}$ Department of Physics, University of Aveiro, 3810-193 Aveiro, Portugal \\ ${ }^{3}$ Department of Glass and Ceramic Engineering, University of Aveiro, CICECO, 3810-193 \\ Aveiro, Portugal \\ amarcia@dq.ua.pt; baspereira@dq.ua.pt; 'a20828@alunos.fis.ua.pt; ${ }^{d}$ kholkin@cv.ua.pt; \\ etita@fis.ua.pt; ${ }^{\mathrm{f}}$ ttrindade@dq.ua.pt
}

Keywords: quantum dots; layer-by-layer methods; semiconductors; nanostructures

\begin{abstract}
Cadmium chalcogenide quantum dots (QD's) were synthesised using a single source approach while zinc oxide QD's were obtained by a colloidal technique. In both situations the dots were surface capped with tri-octylphosphine oxide (TOPO) hence leading to nanodispersed systems in organic solvents such as toluene. The organically capped QD's (CdSe, CdS and ZnO) were used as building-units to fabricate LbL (layer-by-layer) films on glass and quartz substrates. A linear increase in the visible light absorbance (due to the QD's) with the number of deposited layers indicates that multi-layered systems have been fabricated. In order to investigate the effect of the LbL manipulation on the integrity of the QD's, comparative studies on the optical properties of the starting QD's and the nanostructured films have been performed. The observation of quantum size effects in both cases suggests minimal degradation of the QD's though clustering had probably occurred, a point which was further confirmed by AFM analysis.
\end{abstract}

\section{Introduction}

Nanosized particles are of great scientific and technological interest because they have properties different from those of bulk solids of the same composition [1-3]. A range of metals and semiconductor nanocrystals (or QD's) have been prepared using diverse and now well-established chemical methods [1-3]. More recently, intense research has been devoted to the fabrication of nanostructured objects such as ultra-thin films and nanoparticles assemblies [3,4]. New collective properties can result from interactions of the individual nanoparticles when they are organized into such nanostructures. The Langmuir-Blodgett (LB) manipulation, colloid crystallization and layerby-layer (LbL) assembly methods have been the most often used to assemble inorganic nanocrystals into 2D and 3D arrays of nanoparticles [3,4]. All these methods have in common the fact that mesoscopic units have been used as building blocks to fabricate a macroscopic material, hence they belong to the general strategy termed as "bottom-up" approach to materials preparation.

The LbL deposition method consists on the adsorption of alternate layers of oppositely charged polyelectrolytes (PE) on a solid substrate [5,6]. The fabrication of nanostructured objects using this approach has also involved the use of diverse building units such as polyoxometalate anions [7-9], surface coated nanoparticles [3,10] and clays [11]. The multilayers formed by the PE and these building units are stabilized mainly by electrostatic forces, although this type of interactions are not a pre-requisite to fabricate LbL multilayers. Most of the work reported in the literature concerning QD's refers to the deposition of LbL multilayers using charged building-units; surface coated nanoparticles, highly stable in water, have been especially investigated as the starting materials $[3,10]$. It appears that in this case electrostatic interactions play a significant role in the stabilization of the final LbL films. However, no reports have been found in the literature concerning the LbL deposition of organically capped QD's dispersed in an organic medium. In this work TOPO capped QD's, dispersed in toluene, were used as building units to fabricate LbL multilayers. 


\section{Experimental}

Chemicals were readily available from commercial sources (Aldrich) and were used as received without further purification. TOPO capped CdSe and CdS QD's were prepared using the thermolysis of the single molecule precursors $\mathrm{Cd}\left[\mathrm{N}\left(\mathrm{SeP}^{i} \mathrm{Pr}_{2}\right)_{2}\right]_{2}$ and $\mathrm{Cd}\left(\mathrm{S}_{2} \mathrm{CNEt}_{2}\right)_{2}$, in hot TOPO, respectively [12,13]. ZnO and $\mathrm{ZnO}$ :Co QD's were prepared using the method reported by Gamelin et al [14]. Surface modification of the isolated ZnO QD's was performed by treating this material in $\mathrm{TOPO}$, at $150^{\circ} \mathrm{C}$, under a nitrogen stream. Stable and optically clear sols were obtained by dispersing the TOPO capped QD's (CdSe, CdS and ZnO) in toluene. A typical preparation of a LbL multilayer film was carried out using the polyelectrolyte $\mathrm{PAH}$ (polyallylamine hydrocloride) as follows. A cleaned glass or quartz substrate was immersed in a $10^{-2} \mathrm{M}$ PAH aqueous solution over 10 minutes, in order to obtain an homogeneous PAH precursor film. The PE concentration was based on the molecular weight of the monomer unit. Following this deposition step, the film was rinsed with deionized water to remove the material in excess, and then was dry under a nitrogen stream. The substrate-supported precursor film was then alternately dipped into a toluene solution containing the QD's and the PAH solution, in this sequence and during 10 minutes for each deposition. The films were washed using the respective solvent and dried as described above. This process was repeated until the desired number of [QD's/PAH] layers were obtained.

The LbL deposition was monitored by UV/Vis spectroscopy using a Jasco V-560 UV/Vis spectrophotometer. Particle sizes (in diameters) were estimated using the optical spectra following methodologies described in the literature [1-3]. Photoluminescence (PL) was generated using the $325 \mathrm{~nm}$ light from a cw He-Cd laser, with excitation power density less than $0.6 \mathrm{~W} . \mathrm{cm}^{-2}$. The PL was measured at room temperature using a Spex 1704 monochromator $\left(1 \mathrm{~m}, 1200 \mathrm{~mm}^{-1}\right)$ fitted with a cooled Hamamatsu R928 photomultiplier. Atomic force microscopy (AFM) measurements were performed using a NanoScopeIIIa instrument.

\section{Results and discussion}

We have been particularly interested in the synthesis of QD's using single-molecule precursors, i.e. compounds containing both elements of the semiconductor within the same molecule [2,12]. For example, the controlled thermolysis of metal dithiocarbamato or diselenocarbamato complexes in hot TOPO (e.g. $200^{\circ} \mathrm{C}$ ) yields nearly monodispersed metal chalcogenide QD's. Adjusting the reaction temperature, distinct particle size distributions can be obtained, hence allowing band gap tuning. The chemical nature of the surface of QD's synthesised in TOPO have been documented elsewhere $[15,16]$. In brief, the TOPO molecules coordinate to metal sites located at the particles surface, leaving the hydrophobic octyl chains directed outwards. Due to this type of surface, TOPO capped QD's are easily dispersible in a range of organic solvents, including toluene that was used as the solvent in this work. Although TOPO methods have been established as powerful routes to prepare organically capped QD's, the materials obtained by such methods have not been used in LbL assembly studies. We explore the influence of this type of surface on the LbL deposition process via polyelectrolyte-QD interactions and, therefore, the influence of the organically capped building units on the final properties of the as prepared LbL films.

Fig. 1 shows the UV/visible absorption spectra recorded during the LbL deposition of TOPO capped CdSe QD's $(\mathrm{d} \approx 4 \mathrm{~nm}$ ) on a glass substrate. Similar results were obtained for CdS QD's and when quartz was used instead of glass. Fig. 1 clearly shows that the CdSe dots are strongly attached to the glass substrate treated with PAH. As the number of deposition steps increases, the absorbance due to the presence of the QD's also increases, indicating that the QD's have been gradually adsorbed onto the surface formed by PAH. Note that inset in Fig. 1 shows an almost linear variation for a plotting of absorbance at $515 \mathrm{~nm}$, corresponding to the CdSe QD's excitonic peak, in function of the number of deposition steps, suggesting an almost constant mass transference from the solutions to the substrate. The band profile in the optical spectra shown in Fig. 1 is similar to that of the starting QD's dispersed in toluene. TOPO capped QD's exhibit optical properties as a function of their size, in this case the optical band edge of CdSe QD's is blue-shifted from the bulk band edge $(\mathrm{Eg}=1.75 \mathrm{eV})$ due to strong $3 \mathrm{D}$ quantum confinement effects. Therefore the optical properties 
observed in Fig. 1 for the LbL films proceed directly from the unique properties of the constituent CdSe QD's. Additionally this also indicates that the CdSe QD's in the final LbL films retain their integrity due to the surface cap formed by the TOPO molecules.

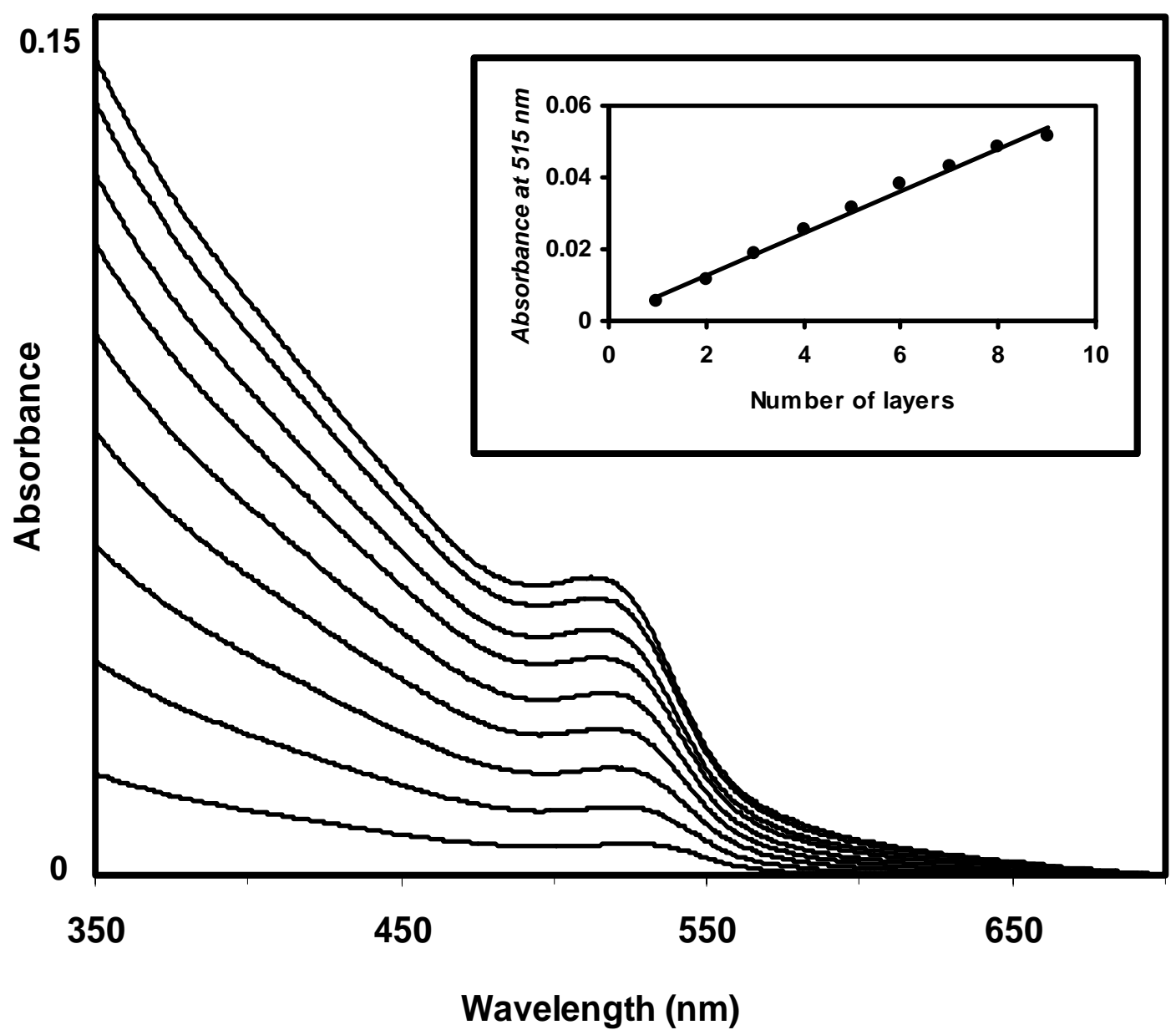

Figure 1: UV/Vis absorption spectra of the sequential steps during the formation of TOPO-capped CdSe QD's/PAH multilayers, from 1 (bottom) to 9 (top). Inset: absorption at $515 \mathrm{~nm}$ vs. number of layers.

In order to confirm that LbL procedures can be used to deposit TOPO QD's, the above procedures were applied to QD's which were prepared using a distinct synthetic approach. For this purpose, pure and Co(II) doped ZnO QD's were chosen as the building units. The ZnO QD's were synthesised following the general strategy described by Gamelin et al. to produce diluted magnetic semiconductors (DMSs) [14]. Prior to the employment of LbL procedures, the ZnO QD's were surface modified by treatment in hot TOPO under a $\mathrm{N}_{2}$ stream. This surface treatment led to slightly larger QD's, as reported previously [14]. Infrared spectroscopy was used to monitor this surface exchange reaction. Fig. 2 shows the UV/Vis absorption spectra of the sequential steps during the formation of TOPO-capped ZnO:Co QD's/PAH multilayer films. Similarly to the LbL deposition of the cadmium chalcogenide QD's (Fig. 1), as the number of deposition steps increases, the absorbance due to the presence of the TOPO capped ZnO:Co QD's also increases, indicating that the QD's have been gradually adsorbed onto the surface formed by PAH and retain their initial morphological characteristics. The optical features observed in Fig. 2 are typical of ZnO QD's (d $\approx$ $6 \mathrm{~nm}$ ). These quantum size effects are clearly noticed considering the slight blue shift observed in the optical band gap in relation to the $\mathrm{ZnO}$ bulk band gap $(\mathrm{Eg}=3.3 \mathrm{eV})$. The final TOPO capped ZnO:Co QD's (blue coloured) also show in the visible region $(500-750 \mathrm{~nm})$ the typical absorption of $\mathrm{Co}(\mathrm{II})$, in the tetrahedral coordination environment in $\mathrm{ZnO}$ (inset in Fig.1) [14]. This optical 
feature results from d-d transitions and is too weak to be observed on the same sensitivity scale, when compared to the $\mathrm{ZnO}$ band gap transition.

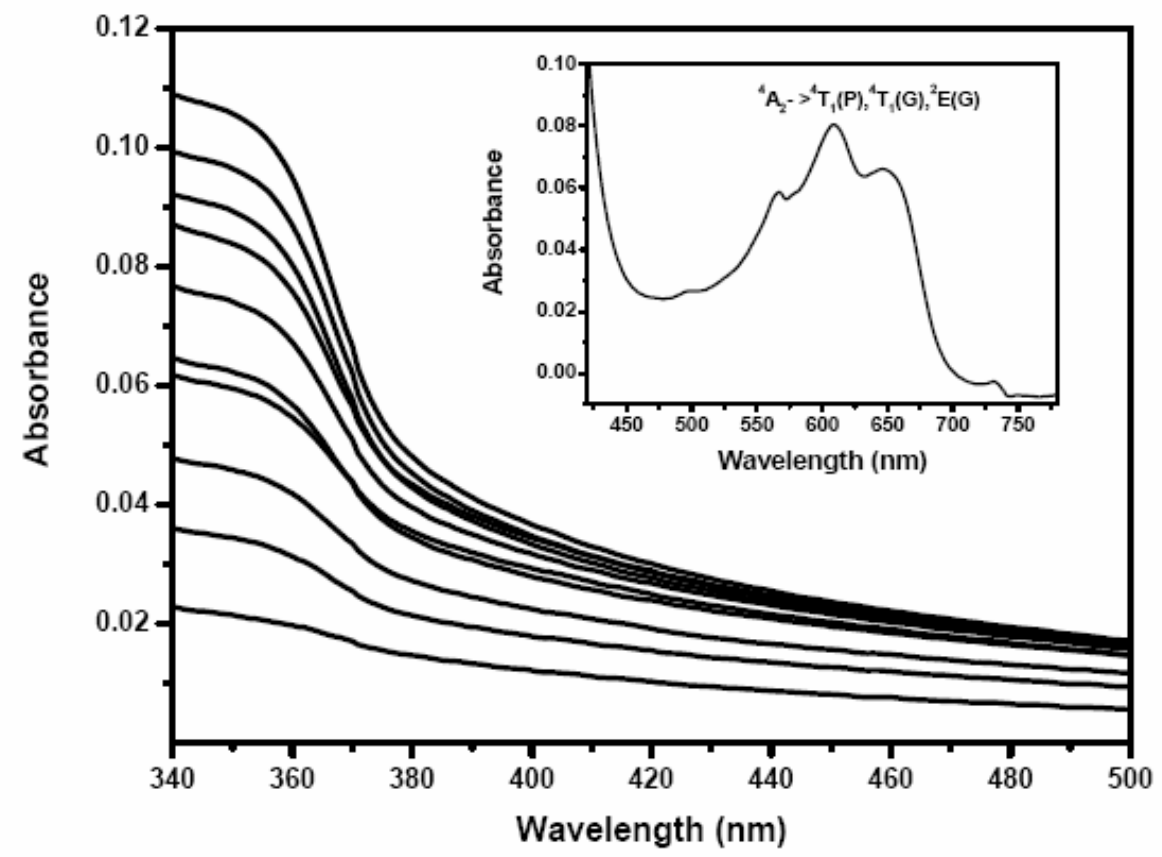

Figure 2: UV/Vis absorption spectra of the sequential steps during the formation of TOPO-capped ZnO:Co QD's/PAH multilayer films, from 1 (bottom) to 10 (top). Inset: visible absorption spectrum of the $\mathrm{ZnO}$ :Co colloid.

Associated to its simplicity, one of the important characteristics of LbL manipulation is the possibility of fabrication of well organised nanostructured materials. Attempts to characterize the morphological properties of the organically capped QD's/PAH layers described above have been made using AFM techniques. Fig. 3 shows an AFM image of a layer of TOPO capped CdSe QD's deposited on a glass substrate using the PE PAH. Well aligned nanostructures around 10-15 nm diameter are clearly visible. Considering that the starting dots have an average size of $4 \mathrm{~nm}$, these larger nanostructures probably correspond to a few number of CdSe QD's wrapped on PAH molecules. Further evidence for this type of arrangement was obtained by PL measurements (not shown) performed on CdSe QD's LbL films obtained from distinct particle size distributions. While the PL emission peak deviates to high energy as the particle size decreases, in agreement with the presence of quantum size effects due to the QD's, a broadening on the emission band compared to the original CdSe QD's was also observed. This emission band broadening might be then associated to clustering of the CdSe QD's mediated by the presence of the polyelectrolyte.

\section{Conclusions}

We have demonstrated that organically caped QD's can be used to fabricate LbL multilayers. In our study, hydrophobic QD's dispersed in toluene have been successfully deposited on glass and quartz substrates previously treated with PAH. In the final nanostructured films, the QD's seem to be clustered via polyelectrolyte interactions, though the integrity of the individual dots is maintained. This study supports results obtained by others [17,18] in which other interactions rather than electrostatic attraction may constitute the driving force to fabricate stable LbL films. For the systems reported here (TOPO capped QD's) van der Waals interactions seem to play a critical role in LbL deposition. 


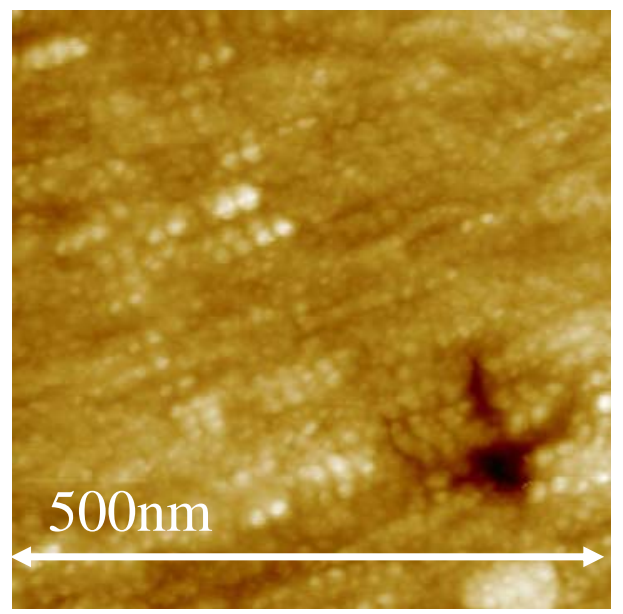

Figure 3: AFM image of a LbL layer of TOPO-capped CdSe QD’s/PAH deposited on glass

\section{Acknowledgements}

ASP (FCT for a PhD grant); funding: University of Aveiro (Project 3.64.33.7/NANOENG/CTS15) and FCT/FEDER (POCTI/CTM/45236/02).

\section{References}

[1] A. Eychmüller: J. Phys. Chem. B Vol. 104 (2000), p. 6514

[2] T. Trindade, P. O’Brien, N.L. Pickett: Chem. Mater. Vol. 13 (2001), p. 3843

[3] L. M. Liz-Marzán, P. V. Kamat (Editors): Nanoscale Materials (Kluwer Academic Publishers; Netherlands 2003).

[4] P. Jiang, J. F. Bertone, V. L. Colvin: Science Vol. 291 (2001), p. 453.

[5] G. Decher, J. Smith: Prog. Colloid Polym. Sci.: Vol. 89 (1992) p.160.

[6] G. Decher: Science Vol. 277 (1997), p. 1232.

[7] S. Liu, D. G. Kurth, B. Bredenkötter, D. Volkmer: J. Am. Chem. Soc. Vol. 124 (2002), p. 12279

[8] S. Liu, Z. Tang, Z. Wang, Z. Peng, E. Wang, S. Dong: J. Mater. Chem. Vol. 10 (2000), p. 2727

[9] F.L. Sousa, A.C.A.S. Ferreira, R. A. Sá Ferreira, A. M. V. Cavaleiro, L. D. Carlos, H. I. S. Nogueira, J. Rocha, T. Trindade: J. Nanosci. Nanotechnol. Vol. 4 (2004), p. 214

[10]N. A. Kotov: MRS Bulletin Vol. 26 (2001), p. 992.

[11]E. R. Kleinfield, G. S. Ferguson: Science Vol. 265 (1994) p. 370.

[12]T. Trindade, P. O’Brien, X. Zhang: Chem. Mater. Vol. 9 (1997) p. 523.

[13]D. J. Crouch, P. O'Brien, M. A. Malik, P. J. Skabara, S. P. Wright: Chem. Commun. Vol. 12, (2003) 1454.

[14]D. A. Schwartz, N. S. Norberg, Q. P. Nguyen, J. M. Parker, D. R. Gamelin: J. Am Chem. Soc. 125 (2003) p.13205.

[15]L. R. Becerra, C. B. Murray, R. G. Griffin, M. G. Bawendi: J. Chem. Phys. Vol. 100 (1994) p. 3297.

[16]J. E. B. Katari, V. L. Colvin, A. P. Alivisatos: J. Phys. Chem. Vol. 98 (1994) p. 4109.

[17]Y. Shimazaki, M. Mitsuishi, S. Ito, M. Yamamoto: Langmuir Vol. 14 (1998) 2768.

[18]E. C. Hao, T. Q. Lian: Langmuir Vol.16 (2000) 7879. 